\section{Polyketide pas de deux}

\section{By Michael J. Haas, Senior Writer}

Two groups have reported methods that overcome key obstacles to studying polyketide biosynthesis in symbiotic and single organisms. Each method advances the exploration of the chemical space for polyketides, which are important for their antibiotic, anticancer, anti-inflammatory and other therapeutic activities, but more experiments are needed to evaluate the full potential of the methods.

One approach used the structures of polyketides produced by bacterial symbionts of marine sponges as a starting point for identifying the corresponding polyketide synthases (PKSs), which are the enzymes that synthesize and assemble ketide structures into polyketide chains and rings. ${ }^{1}$ The second tactic consisted

\section{"The strategy may represent a general, rapid way to target the polyketide assembly lines."}

-Yi Tang,

University of California, Los Angeles involved in the biosynthesis of psymberin, an antitumor polyketide found in the marine sponge Psammocinia aff. bulbosa. They designed a PCR primer sequence to target each predicted PKS gene and used the primers and conventional PCR to amplify the psymberin-producing PKS genes and fish them out of the metagenome pool.

The approach enabled the team to identify all genes involved in psymberin biosynthesis directly from the combined metagenome without having to determine which bacterial species produced the compound. Researchers got results in weeks and used only a few grams of material from the sponge.

The original structural characterization of psymberin, done by a group at the University of California, Santa Cruz, required about 600 extracts from sponge material collected over 11 years. ${ }^{4}$

Jörn Piel, leader of the Bonn team, said the method has broad applications because the trans-acyltransferases are not unique to the bacterial symbionts found in sponges.

"These PKSs have also been discovered by us in a beetle symbiont and by other researchers in symbionts" from a fungus and a species of bryozoan, which is a coral-like marine animal, he said. "We therefore believe that the targeting method can be applied to a wide range of hostsymbiont associations."

Piel is professor of biological and organic chemistry at Bonn's Kekulé Institute of Organic Chemistry and Biochemistry. The team also of coaxing a fungus into expressing ordinarily silent genes in its genome and then identifying any unusual polyketides that resulted. ${ }^{2}$

The research teams said their respective methods should be applicable to polyketides produced in other symbiotic systems or other eukaryotic organisms.

\section{Sponge bag}

Marine sponges can harbor hundreds of bacteria that produce polyketides. But because they live in symbiosis with the sponge, these bacterial species cannot be cultivated individually to determine which PKSs each bacteria expresses or which polyketide structures each produces.

A research team at the University of Bonn developed a way around the symbiosis problem. They backtracked from particular polyketide structures to the corresponding PKS genes in the combined sponge-bacterial genome, the metagenome.

Last year the Bonn team reported that the genetic sequences of trans-acyltransferases-a class of PKSs frequently found in symbiotic bacteria - could be used to predict the specific polyketide structures that the corresponding enzymes produced. ${ }^{3}$ This finding led the team to hypothesize that the correlation could also be used in reverse, to point from a known polyketide structure to the PKSs involved in its biosynthesis.

In a new paper in Nature Chemical Biology, the team described tests on whether this hypothesis could help identify the PKS genes included researchers from University of California, Santa Cruz, the Fritz Lipmann Institute and New Zealand's National Institute of Water and Atmospheric Research Ltd.

\section{Fishing expedition}

Given the multitude of bacterial symbionts in marine sponges and the difficulties in cultivating those individual bacterial species, the Piel team's strategy "may represent a general, rapid way to target the polyketide assembly lines," said Yi Tang, associate professor of chemical and biomolecular engineering at the University of California, Los Angeles.

"For sponges, genetic sequencing of the whole organism and symbionts is difficult, and therefore Piel's team's approach is wonderful," said Nancy Keller, professor of medical microbiology, immunology and bacteriology at the University of Wisconsin-Madison.

Robert Fecik, associate professor of medicinal chemistry at the University of Minnesota, also thinks the method will be broadly applicable to complex sponge-bacterial systems. He noted that the approach used routine microbiology techniques that should be easy for other researchers to adopt, and it focused on structural features common to polyketides produced by symbiotic bacteria, not on a structural feature specific to psymberin.

Given these advantages, he said, "I think it is possible that there are previously unknown polyketides that could be discovered this way, even though the method is not intended to identify novel polyketides." 
For instance, Fecik suggested that the method could be modified to fish out of the metagenome all PKS genes that produce a structural feature shared by multiple polyketides. Those results could serve as a starting point to find previously unknown biosynthetic pathways and the polyketides they produce.

Tang did say that despite the two encouraging examples, the strategy was still a trial-and-error approach that could require testing many designed primers before isolating the desired gene product. "Additional successful fishing examples using this strategy are needed to further demonstrate the efficiency of this approach and support its wide application," he said.

Both Tang and Fecik said that follow-on experiments would be needed to confirm the functions of the putative psymberin-producing genes by expressing them in Escherichia coli.

Piel said his team had already begun these experiments.

\section{The fungus speaks}

Meanwhile, the study of polyketide biosynthesis in nonsymbiotic organisms presents its own challenges. Many microorganisms harbor nontranscribed (silent) gene clusters that genetic sequencing suggests code for PKSs and other enzymes involved in natural product biosynthesis. As long as the genes remain silent, any currently unknown polyketides they might produce remain unknown.

The regulatory mechanisms underlying the silencing process are not well understood, but studies in the past two years have suggested that regulation at the chromatin level can be responsible for keeping the genes quiet. ${ }^{5,6}$

Based on this, a team led by Keller and Clay Wang, assistant professor of pharmaceutical sciences and chemistry at the University of Southern California, sought to determine whether modifying chromatin-regulating processes such as methylation could induce a fungus-Aspergillus nidulans - to express its silent genes and thereby produce additional polyketides. ${ }^{2}$

Indeed, when the group knocked out a histone 3 methyltransferase in the fungus, the organism synthesized polyketides it had not previously produced.

Among those compounds were eight polyketides with known biological activity: the anthraquinones monodictyphenone and emodin plus four emodin analogs, which all have anticancer and anti-inflammatory activity, and two polyketides, F9775A and F9775B, which have antiosteoporosis activity.

Keller said the team's method should work in any eukaryote that harbors silent gene clusters and thus could extend to other organisms that produce biologically active compounds, such as alkaloid-producing plants.

However, Sarah O'Connor, associate professor of chemistry at the Massachusetts Institute of Technology, cautioned that it was not yet clear whether the method was applicable to alkaloid-producing plants. "Rational manipulation of chromatin regulation in plants is difficult, and unpredictable results have resulted from many efforts in plant systems," she said. "However, I certainly think that this strategy would be worth a try in a more complicated eukaryotic organism such as a plant."

\section{Silence management}

Natural products researchers agreed that the Keller and Wang team's method overcame a major challenge in natural product discovery-the expression of silent metabolite gene clusters under laboratory conditions to produce new polyketides.

"Silent gene clusters have been found in many types of microorganisms that produce polyketides," Fecik said. By addressing the challenges involved in turning on these genes, he said the Keller and Wang team's method has general applicability to natural product research, especially because the techniques involved do not require specialized knowledge.

Tang agreed that the method was a big step forward in tapping the polyketide-producing power of silent genes. "This paper demonstrated for the first time the feasibility of simple chromatin landscape modification in expression of silent fungal secondary metabolite gene clusters," he said.

Tang said the approach would be even more exciting if the team eventually finds that the modified $A$. nidulans produced previously unknown polyketide scaffolds in addition to the eight known polyketides reported in the paper. He said a full exploration of the method's potential would require studies of other chromatin-regulating mechanisms besides the silencing of histone 3 .

Other members of the Keller and Wang team included researchers from Taiwan's Chia Nan University of Pharmacy and Science, The Ohio State University, Austrian Research Centers GmbH and the University of Natural Resources and Applied Life Sciences.

\section{Going broader}

Besides confirming the functions of the individual psymberin-related enzymes in vitro, Piel said his team is working to express the full psymberin biosynthetic pathway in E. coli to produce larger amounts of psymberin than are available from the natural source.

The University of Bonn has filed a patent application for the genetargeting method and the psymberin genes reported by Piel's team in the Nature Chemical Biology paper. "We are currently looking for industrial partners," Piel told SciBX.

Keller and Wang are working to identify additional polyketides produced by their modified $A$. nidulans. "Future studies will include antimicrobial and anticancer assays of the identified compounds," Keller said.

She added that the findings her team reported in Nature Chemical Biology are not patented or licensed.

"It is reasonable to expect that the methods described in both papers will have broad applicability, not just to the specific organisms studied in each case, but also in how widely the methods can be applied to other organisms," said Fecik.

Haas, M.J. SciBX 2(22); doi:10.1038/scibx.2009.898

Published online June 4, 2009

\section{REFERENCES}

1. Fisch, K. et al. Nat. Chem. Biol.; published online May 17, 2009; doi:10.1038/nchembio.176 Contact: Jörn Piel, University of Bonn, Bonn, Germany e-mail: joern.piel@uni-bonn.de 
2. Bok, J. et al. Nat. Chem. Biol.; published online May 17, 2009; doi:10.1038/nchembio.177

Contact: Nancy P. Keller, University of Wisconsin-Madison, Wis. e-mail: npkeller@wisc.edu

3. Nguyen, T. et al. Nat. Biotechnol. 26, 225-233 (2008)

4. Cichewicz, R. et al. Org. Lett. 6, 1951-1954 (2004)

5. Shwab, E. et al. Eukaryot. Cell 6, 1656-1664 (2007)

6. Roze, L. et al. Mol. Microbiol. 66, 713-726 (2007)

COMPANIES AND INSTITUTIONS MENTIONED

Austrian Research Centers GmbH, Vienna, Austria

Chia Nan University of Pharmacy and Science, Tainan, Taiwan
Fritz Lipmann Institute, Jena, Germany

Massachusetts Institute of Technology, Cambridge, Mass.

National Institute of Water and Atmospheric Research Ltd.,

Wellington, New Zealand

The Ohio State University, Columbus, Ohio

University of Bonn, Bonn, Germany

University of California, Los Angeles, Calif.

University of California, Santa Cruz, Calif.

University of Minnesota, Minneapolis, Minn.

University of Natural Resources and Applied Life Sciences,

Vienna, Austria

University of Southern California, Los Angeles, Calif.

University of Wisconsin-Madison, Madison, Wis. 